\title{
Impacto econômico da mosca dos chifres em bovinos de corte
}

\author{
Horn fly economic impact in beef cattle
}

\author{
Michael Allim Jorge ${ }^{1}$; Cristiene Rosa²; Glauber dos Santos ${ }^{3 *}$
}

\author{
${ }^{1}$ Universidade Anhembi Morumbi - Graduando em Medicina Veterinária - Rua Dr. Arnaldo de Almeida Lima, \\ 1134 - Parque da Moca - CEP 03164-000 - São Paulo (SP), Brasil \\ 2 Universidade Anhembi Morumbi - Doutora em Medicina Veterinária - Rua Dr. Arnaldo de Almeida Lima, 1134 \\ - Parque da Moca - CEP 03164-000 - São Paulo (SP), Brasil \\ 3 Instituto PECEGE - Doutor em Zootecnia - Rua Alexandre Herculano,120 - T6 - Vila Monteiro - CEP 13418- \\ 445 - Piracicaba (SP), Brasil
}

\section{Resumo}

A mosca dos chifres (Haematobia irritans) é um dos principais ectoparasita de bovinos na pecuária atual no Brasil, por isso objetivou-se avaliar o impacto econômico deste parasita em relação ao ganho de peso e a idade para o abate em bovinos de corte. O estudo foi baseado na pesquisa de Bianchin et al. (2004), onde realizaram um estudo na região do cerrado de Mato Grosso do Sul, durante a estação chuvosa de outubro a maio, época de maior infestação do parasito. Foram utilizados os dados de 20 machos com idade de dois anos, castrados da raça nelore e de mesmo peso inicial, divididos em dois grupos de dez animais tratados com inseticidas $\mathrm{x}$ animais não tratados. Foram avaliados o ganho de peso e a idade de abate para determinar a eficiência e o impacto econômico de cada grupo. O desempenho animal apresentou um acréscimo de $16 \%$ no ganho de peso diário e redução de $32 \%$ em relação a idade de abate de animais tratados com antiparasitário. Em tais condições, o impacto econômico positivo foi de $R \$ 154,64$ por animal. Os resultados evidenciaram que se justifica o tratamento do antiparasitário, pois a eficiência na redução da mosca dos chifres proporcionou um impacto econômico positivo para o sistema de produção de gado de corte.

Palavras-chave: desempenho animal, ganho de peso, gestão rural, Haematobia irritans

\section{Abstract}

The horn fly (Haematobia irritans) is one of the main ectoparasite of cattle in the current livestock farming in Brazil, so we aimed to evaluate the economic impact of this parasite in relation to weight gain and age of slaughter of beef cattle. The study is based on Bianchin et al. (2004) research, who conducted a study in the state of Mato Grosso do Sul, in Cerrado region, during the rainy season from October to May, when is the greatest time of the parasite infestation. The data used in the study was 20 two-year-old males of the breed Nelore, all castrated and with the same initial weight, divided into two groups, one of ten animals treated with insecticides and another with ten untreated animals. They evaluated the weight gain and age of slaughter to determine the efficiency and the economic impact of each group. The animal performance was $16 \%$ higher in the daily weight gain and the age of slaughter was $32 \%$ lower for animals treated with the antiparasitic. In such conditions, it had a positive impact of $\mathrm{R} \$ 154.64$ per treated animal in the economy. The results justify the treatment with the insecticide, as the efficient reduction of the horn fly provided a positive economic impact for beef cattle production system.

Keywords: animal performance, weight gain, rural management, Haematobia irritans

\footnotetext{
${ }^{1}$ Autor correspondente < glauber@pecege.org.br> Enviado: 05 maio 2016

Aprovado: 30 maio 2016
} 


\section{Introdução}

A mosca dos chifres (Haematobia irritans), descrita por Linnaeus em 1758, foi reconhecida como uma praga de bovinos na França em 1830 e foram identificadas primeiramente no Brasil no estado de Roraima provavelmente vindo da Guiana por volta de 1977, com seu primeiro registro feito por (Valério e Guimarães, 1983).

Segundo Bianchin e Alves (2002), este inseto se encontra nas partes do animal que ficam fora do alcance do movimento da cabeça e cauda do animal (cupim, costa, barriga e pernas). Ainda segundo o mesmo autor, mosca dos chifres exibe preferência para bovinos de raça europeia, mestiço e animais de pelagem escura ou com manchas escuras e para machos inteiros. No entanto, a ocorrência da atividade hematófaga da mosca dos chifres não é seu aspecto mais nocivo, mostram em seus estudos que o acometimento das picadas dolorosas e incessantes durante todo o dia sobre os bovinos parasitados são os aspectos de maiores importâncias em suas ações (Brito et al., 2005).

O Brasil tem aproximadamente 212,3 milhões de bovinos, segundo o Instituto Brasileiro de Geografia e Estatística (IBGE, 2014), portanto o parasita representa um grande potencial de impacto negativo para atividade pecuária atual (Stotzer et al., 2014). Sendo que a mosca encontra condições favoráveis na maior parte do país, onde o seu ciclo no período chuvoso pode se completar em 8-12 dias (Honer e Gomes, 1990) e pode apresentar até 19,1 gerações anuais (Bianchin et al., 2006), comprometendo muitas vezes o processo de transformação dos fatores de produção em produtos de origem animal.

Além das condições mencionadas acima, a crescente utilização de cruzamentos entre zebuínos (Bos taurus indicus) e taurinos (Bos taurus taurus) como intuito de associar adaptação com a produção de carne bovina como relatado por (Paulino et al., 2014) vem aumentando a exposição dos bovinos ao inseto. De acordo com Honer et al., (1990) os Bos indicus são menos susceptíveis à infestação por moscas do que os Bos taurus, logo quanto menor a proporção de sangue zebuíno, maior a infestação por mosca dos chifres incitando a elevação do custo de produção afetando diretamente a produtividade da pecuária brasileira.

Os danos atribuídos à $H$. irritans com respeito ao rebanho brasileiro de bovinos foram estimados por Grisi et al. (2014) em US\$2,56 bilhões anuais. Os prejuízos desta estão relacionados a transmissão de patógenos (carbúnculo hemático, leucose, anaplasmose e do helminto Stephanofilaria sp, também das miíases). Além da veiculação de patógenos o parasita gera estresse ao animal, pois este tenta se livrar das moscas e acaba se debatendo muito. Tal fato, contribui para um elevado gasto energético, diminui o 
tempo de pastejo e ingestão de água, retardando assim o ganho de peso o qual reflete no aumento da idade para o abate dos bovinos de corte (Bianchin e Alves, 2002; Brito et al., 2005). Diante deste cenário de danos, uma análise econômica de estratégias de controle se faz necessário, para conhecer os custos e benefícios do controle a este parasita.

É através da análise econômica que o produtor passa a conhecer cada atividade da empresa rural, podendo assim concentrar esforços nos principais pontos de estrangulamento do processo produtivo (Lopes e Carvalho, 2002; Ziliotto et al., 2010; Lopes et al., 2012). Mediante esses resultados econômicos que o gestor pode tomar, conscientemente, suas decisões com o intuito de conduzir o seu sistema de produção de gado de corte como uma empresa, abandonando a posição de amadorismo e passando a empreendedor.

Assim dada a importância do crescimento desse parasito em termos de infestação no rebanho nacional de gado de corte, objetivou-se avaliar os impactos econômicos da mosca dos chifres em relação ao ganho de peso e a idade para o abate em bovinos de corte, baseado em dados de desempenho animal de Bianchin et al. (2004).

\section{Metodologia}

Os dados de desempenho animal foram baseados na pesquisa de Bianchin et al. (2004), onde realizaram um estudo durante a estação chuvosa de outubro a maio totalizando 240 dias, época de maior infestação de $H$. irritans nos animais. Foram utilizados os dados de 20 machos castrados da raça nelore que continham dois anos de idade e de mesmo peso vivo inicial.

Todavia a metade dos animais foram tratados a cada 28 dias com inseticida do grupo Piretroide, princípio ativo Deltametrina. Assim sendo, foram utilizados dois tratamentos (tratados com inseticidas $x$ animais não tratados) (Tabela 1). $E$ a partir daí, a cada 14 dias, foram realizadas a contagens do número de mosca dos chifres em um dos lados e o resultado multiplicado por dois, foi também efetuada, a cada 28 dias, a pesagem dos animais. 
Tabela 1. Peso inicial, final e ganho de peso médio diário [GMD] dos animais em função de serem tratados ou não

\begin{tabular}{cccc}
\hline Tratamento & Peso inicial & Peso final & GMD \\
\hline Tratados & 225,9 & 375,8 & 0,620 \\
Não tratados & 221,6 & 345,5 & 0,520 \\
\hline
\end{tabular}

Fonte: adaptado de Bianchin et al. (2004)

O ganho de peso médio diário [GMD] foi resultante do ganho de peso dividido pelo período de tratamento (240 dias). Em função do GMD estimou-se o tempo necessário para os animais atingirem o peso para o abate, esse sugerido aqui como sendo de $510 \mathrm{~kg}$ de peso vivo (17 arrobas).

Para estimar o custo para os animais atingirem o peso de abate, foram utilizados 0 tempo a mais que o animal permanecia no campo para atingir o peso sugerido de $510 \mathrm{~kg}$ de peso vivo, o que para os animais tratados foi de 218 dias e para os não tratados foi de 319 dias. De posse desse período, multiplicou-se os mesmos pelo custo da diária a mais no campo, esse valor diário foi de $R \$ 1,54$ com base em Ziliotto et al. (2010). O impacto foi considerado como sendo a diferença entre o custo total dos animais tratados para os não tratados.

Foram desenvolvidos dois cenários para projeções de impacto econômico, variando o número de animais: o primeiro com diferentes ganhos de peso adicional, sendo eles: $50 \%$ menor $(0,050 \mathrm{~kg}) ; 50 \%$ maior $(0,150 \mathrm{~kg}) ; 100 \%$ maior $(0,200 \mathrm{~kg}) ; 150 \%$ maior $(0,250 \mathrm{~kg})$ e $200 \%$ maior $(0,300 \mathrm{~kg})$, com relação ao resultado obtido por Bianchin et al. (2004). Tais oscilação foram em função da produtividade nas pastagens, sanidade dos animais e genética dos animais.

O segundo foi contemplando a variação no custo de diárias a partir de $R \$ 1,54$ (Ziliotto et al., 2010), sendo uma variação com queda de $2,60 \%(R \$ 1,50)$ e as demais superiores em 3,90\% ( $R \$ 1,60) ; 6,49 \%(R \$ 1,64) ; 10,39 \%(R \$ 1,70)$ e $12,99 \%(R \$ 1,74)$. Tais variações foram em função de um possível aumento no preço dos insumos (despesas variáveis como medicamentos veterinários, suplemento mineral, óleo diesel, energia elétrica entre outros).

\section{Resultados e Discussão}

Os resultados obtidos mostraram que animais que receberam o tratamento com antiparasitário, aliada ao controle estratégico com piretroides, registraram $0,100 \mathrm{~kg}$ a mais 
de ganho de peso durante o período analisado (outubro até maio), conforme dados apresentados na tabela 1.

Essa diferença representou $30,3 \mathrm{~kg}$ no final do período de estudo. A mosca do chifre contribuiu para aumentar o estresse animal, esse aumentou o gasto energético do animal, fator limitante para que o animal possa obter bons desempenhos (Bianchin e Alves, 2002).

Esses $30,3 \mathrm{~kg}$ representaram duas arrobas, considerando o atual preço de mercado $R \$ 145,00$, o produtor já teria um aumento de receita de $R \$ 290,00$. O mesmo foi verificado por Honer e Gomes (1990) e Honer et al. (1990), no centro oeste, em relação à um bovino com infestação da mosca dos chifres sofreria uma perda de peso de 0,109 kg por dia, aproximadamente, resultado semelhante aos deste estudo.

Na tabela 2 foram apresentados os dados do impacto econômico da estratégia de combate ao parasito. O custo para atingir a idade ao abate dos animais tratados com 0 inseticida foi de $32 \%$ menor, quando comparado com os animais não tratados (tratados $R \$$ 336,07 X não tratados $R \$ 490,71$ ). Tal fato propiciou que os animais tratados tivessem um ganho de peso adicional diário $0,100 \mathrm{~kg}$ sendo $16 \%$ a mais, em comparação aos não tratados.

Tabela 2. Tempo para abate, idade final em meses, total de rentabilidade após abate e impacto econômico dos animais tratados e não tratados

\begin{tabular}{cccc}
\hline Tempo para Abate & Idade final & Total & Impacto Econômico \\
\hline --- dias --- & --- meses --- & $----\mathrm{R} \$$---- & $------\mathrm{R} \$$------ \\
T 218,23 & 38,97 & 336,07 & $* 154,64$ \\
NT 318,64 & 42,32 & 490,71 & \\
\hline
\end{tabular}

Nota: $\mathrm{T}=$ tratados; NT não tratados; * valor por animal

Fonte: Resultados originais da pesquisa

Em relação ao tempo para o abate dos animais tratados (Tabela 2) notou-se que os animais chegaram ao peso de abate mais cedo, com 38 meses e 9 dias de idade. No entanto, houve uma diferença significativa em relação ao total de custo, que foi de $R \$$ 336,07 para os tratados em relação com os não tratados que foi de $R \$ 490,71$. Tal fato resultou em um impacto positivo de $\mathrm{R} \$ 154,64$ por animal. O mesmo foi verificado por Soutello (2001) em estudo comparativo os animais tratados com antiparasitário demostraram maior rentabilidade econômica (2,98\% tratados $X 1,96 \%$ não tratados).

Portanto, com um investimento de $\mathrm{R} \$ 1,68$ por animal com o tratamento antiparasitário associado ao controle estratégico da mosca do chifre foi possível obter uma 
economia de 154,64 por animal. Devido apenas a diferença no ganho de peso diário, o qual reduziu o período necessário para que o animal atingisse o peso sugerido para o abate, de $510 \mathrm{~kg}$ de peso vivo. Tal fato permitiu ao produtor que o animal fosse comercializado em épocas estratégicas proporcionando melhor preço de venda e maior impacto positivo para o setor pecuário. Confirmando Soutello (2001), os animais tratados com antiparasitário, chega mais cedo a idade de abate em épocas estratégicas, ocasionando melhor rentabilidade para o produtor.

$\mathrm{Na}$ tabela 2, observando-se o tempo de utilização de pastagem pelos animais tratados, notou-se que, além do impacto econômico positivo de $R \$ 154,64$ por animal, houve uma permanência dos animais nas pastagens de 3 meses e 3 dias a menos. Tal fato permitiu que o produtor utilize o fator tempo para maximizar o seu sistema de produção, como por exemplo, renovação de pastagens degradadas, rotação das pastagens com o objetivo de redução da compactação de solo, vedação, correção da acidez do solo e adubação, rotação de culturas entre outras. Segundo Balbinot Junior et al. (2009), o sistema de produção que utilizou intensivamente os recursos disponíveis do agrossistema gerou maior renda por área da propriedade.

Desta forma, pode-se observar que os animais não tratados tiveram um desempenho animal significativamente menor que o grupo tratado no presente estudo. Notou-se que, devido ao GMD menor de 0,100 kg por dia, houve um acúmulo de perda de peso de 30,30 kg durante o período de estudo. Tal fato segundo Bianchin et al. (2004), ocorreu devido ao grau médio de infestação da mosca dos chifres de 15 até 34 moscas por animal, sendo inferior à Honer e Gomes (1990); Honer et al. (1990) e Steelman et al. (1991) que descreveram o limiar econômico de aproximadamente 200 moscas por animal, o que representou uma perda de peso anual por animal de $15 \mathrm{~kg}$ ou um prejuízo de $\mathrm{R} \$ 145,00$. Entretanto, considerando que há outros princípios químicos utilizados no tratamento de outros parasitos (endoparasitas e carrapaticidas) que também agem sobre a mosca dos chifres reduzindo a infestação do parasito no animal, fato que colaborou para o animal obter maior ganho de peso. Além disso, o controle biológico com o besouro Onthophagus gazella, que segundo Sant' Anna (2007) e Cicote et al. (2009) podem reduzir em até 40\% a infestação da $H$. irritans presentes no local deste estudo, bem como, os animais da raça nelore serem mais resistentes ao parasito. Tais fatos que não foram considerados no presente estudo, pode ou não, estar diretamente relacionado com a baixa infestação do parasita e ter proporcionado maior ganho de peso para os animais tratados em relação aos não tratados. 
Além da questão econômica, o uso de drogas antiparasitárias devem ser pautas em cuidados afim de evitar resistência nos parasitas. Tal fato de acordo com Bruhn et al. (2012), os parasitos adultos resistentes continuam a eliminar ovos em níveis elevados mesmo após a aplicação dos antiparasitários. O uso intensivo dos piretroides sintéticos desenvolvem resistência aos parasitas em todo território brasileiro. Fato que durante o período de tratamento, a resistência parasitária à piretroide, utilizada neste estudo, parece não ter contribuído para a baixa eficiência (Belo et al., 2012; Barros et al., 2013).

Pode-se afirmar, que para os animais não tratados, a não utilização de antiparasitário resultou em um significativo impacto econômico negativo de $\mathrm{R} \$ 154,64$ por animal. Confirmando o resultado de que animais não tratados, resultaram em um menor nível de proteção aos parasitas (Honer et al.,1990; Bianchin e Alves, 2002; Bianchin et al., 2004; Grisi et al. 2014).

Porém, Honer et al. (1990), Bianchin et al. (2006) e Barros et al. (2014) relataram que a aplicação do controle estratégico, sabendo que as perdas foram mais relevantes na época chuvosa (outubro a maio na região do estudo), ameniza as perdas do desempenho animal, sem, no entanto, proporcionar resistência ao antiparasitário. Tal fato pode, em parte, explicar a menor perda de peso para os animais tratados, bem como, o controle estratégico mais efetivo sobre o parasita. De acordo com Barros et al. (2013), a adoção de controle que reduzem o uso intensivo (descontrolado) do antiparasitário foi a melhor alternativa para um controle efetivo e sustentável.

Através desta projeção observou a influência que o GMD adicional dos animais tratados em relação os não tratados, ou seja, com o aumento do GMD adicional, menor foi o período para o animal atingir o peso de abate e consequentemente menor custo ao abate (Tabela 3). Tal fato, deveu-se ao fator peso para abate ser constante $(510 \mathrm{~kg}$ ), assim um menor ganho de peso diário multiplicado pelo custo diário do animal, maior foi o custo até o animal atingir o peso de abate. Deste modo, as estratégias de reduzir o custo diário ou a diferença de ganho de peso entre os tratamentos tornaram-se coerentes. Os bezerros desmamados com controle antiparasitário apresentaram maior desempenho animal $(8,100$ $\mathrm{kg}$ a mais) em comparação a bezerros desmamados sem o controle de parasita (Steelman et al., 1991)

Outro fato que pode ser observado, foi que o animal tratado com GMD adicional de $0,250 \mathrm{~kg}$ apresentou um impacto positivo de $\mathrm{R} \$ 292,11$ (Tabela 3). No entanto, ao reduzir o GMD adicional para $0,100 \mathrm{~kg}$ observou-se que o impacto também reduz para $R \$ 154,64$. 
Tal diferença referiu apenas para 1 animal, multiplicando por 100 animais o impacto positivo foi de $R \$ 15.464,00$ e quando multiplicado por 1000 animais o impacto positivo foi de $R \$$ 154.640,00. Deve-se lembrar que todas as demais variáveis permanecem constante. 0 impacto econômico causado pela mosca dos chifres no rebanho brasileiro foi de US\$2,6 bilhões (Grisi et al., 2014).

$\mathrm{Na}$ tabela 4, através desta projeção foi possível entender que quanto maior for seu custo da diária maior foi o impacto positivo, ou seja, os animais apresentaram uma menor despesas até atingirem o peso de abate. Tal fato, ocorreu devido ao peso para o abate (510 $\mathrm{kg}$ ), tempo restante para o abate (218 dias após ao termino de tratamento) e GMD adicional de $0,100 \mathrm{~kg}$ variáveis estiveram constantes, assim um maior custo da diária multiplicado pelo número de animais tratados, maior foi o impacto positivo após o animal ser abatido. Observou-se que, quando houve possibilidade de o animal expressar seu maior desempenho, o produtor aumentou a sua produtividade, portanto, obtendo maior retorno financeiro sobre o capital investido. Lopes et al. (2012) descreveram que uma das formas de obter maior rentabilidade foi aumentando a produtividade da propriedade.

$\mathrm{Na}$ tabela 4 observou as variações do preço da diária, considerando todas as outras variáveis fixas, comparado ao número de animais. Notou-se que, com uma diária de $\mathrm{R} \$$ 1,60 obteve impacto positivo de 160,67 por animal tratados e no momento em que a diária passou para $R \$ 1,70$ o impacto positivo foi $R \$ 170,71$ por animal tratado. Por tanto, ao multiplicar por 500 animais tratados conteve um impacto positivo de $R \$ 85.355,52$, ou seja, o produtor deixou de gastar este valor em seu ciclo de produção. 
Tabela 3. Cenário com variações no ganho de peso médio adicional e da quantidade de animais

\begin{tabular}{|c|c|c|c|c|c|c|}
\hline \multirow[t]{2}{*}{ N. de animais } & \multicolumn{5}{|c|}{ Ganho médio diário adicional (kg) } & \multirow[b]{2}{*}{0,300} \\
\hline & 0,050 & 0,100 & 0,150 & 0,200 & 0,250 & \\
\hline 1 & 87,66 & 154,64 & 207,31 & 252,65 & 292,11 & 326,76 \\
\hline 10 & 876,60 & $1.546,40$ & $2.073,10$ & $2.526,10$ & $2.921,12$ & $3.267,58$ \\
\hline 50 & $4.383,01$ & $7.732,00$ & $10.365,50$ & $12.632,50$ & $14.605,59$ & $16.337,91$ \\
\hline 100 & $8.766,02$ & $15.464,00$ & $20.731,00$ & $25.265,00$ & $29.211,19$ & $32.675,82$ \\
\hline 500 & $43.830,10$ & $77.320,00$ & $103.655,00$ & $126.325,00$ & $146.055,93$ & $163.379,10$ \\
\hline 1000 & $87.660,21$ & $154.640,00$ & $207.310,00$ & $252.650,00$ & $292.111,86$ & $326.758,78$ \\
\hline
\end{tabular}

Fonte: Resultados originais da pesquisa

Tabela 4. Cenário com variações no preço da diária e da qualidade de animais

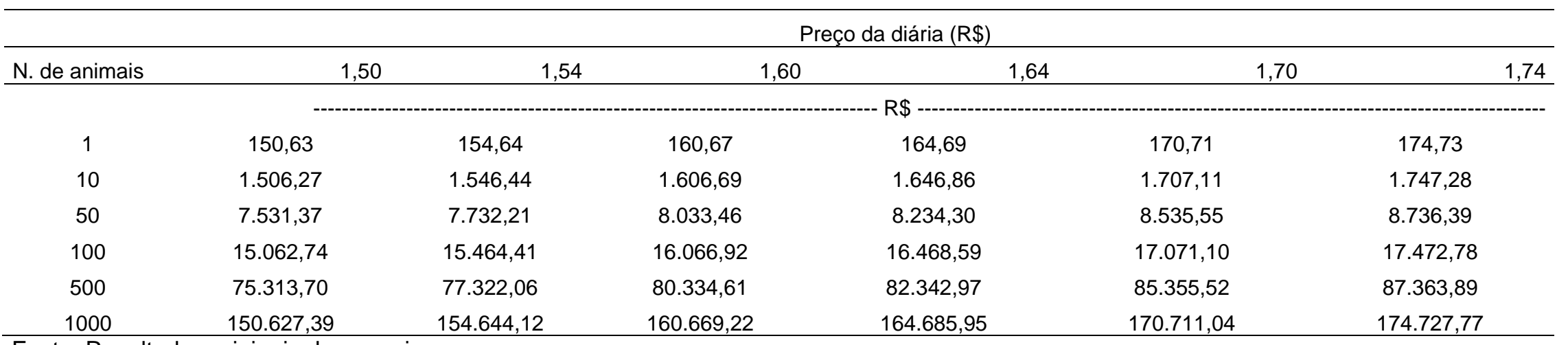

Fonte: Resultados originais da pesquisa 
Mesmo um pequeno produtor com 50 animais tratados com uma diária de $R \$ 1,64$, obteve um impacto positivo de $\mathrm{R} \$ 8.234,30$ (Tabela 4). Tal fato foi de extrema importância, pois apesar de ter a menor representatividade nas pequenas propriedades, a falta de controle do parasito pode, segundo Honer et al. (1990), gerar perdas estimadas de desempenho animal que chegaram a $14 \%$ para novilho de corte e $25 \%$ para a produção de leite. Dessa forma, a divisão em grupos dos animais permitiu ao técnico e ao produtor uma análise mais detalhada do sistema de produção possibilitando detectar mais facilmente os pontos de estrangulamento (Lopes et al., 2009).

\section{Conclusão}

Os animais que recebem antiparasitário obtivem melhor desempenho, atingindo em menor período ao peso de abate, consequentemente proporcionam melhor produtividade e impacto econômico positivo para o sistema de produção.

O tratamento com antiparasitário associado com o controle estratégico nos meses de outubro até maio é o mais adequado para o controlar mosca dos chifres e melhorar a rentabilidade da atividade.

\section{Referências}

Barros, A.T.M. de; Ravaglia, E.; Petzold, H.V.; Avellar, W. de. 2014. Avaliação da estratégia de tratamento parcial do rebanho no controle da mosca dos chifres. (Boletim de Pesquisa e Desenvolvimento / Embrapa Pantanal,125). Embrapa Pantanal, Corumbá, MS. Disponível em:<http://www.cpap.embrapa.br/publicacoes/online/BP125.pdf>. Acesso em: 30 abr. 2016.

Barros, A.T.M.; Schumaker, T.T.S.; Koller, W.W.; Klafke, G.M.; Albuquerque, T.A.D.; Gonzalez, R. 2013. Mechanisms of pyrethroid resistance in Haematobia irritans (Muscidae) from Mato Grosso do Sul state, Brazil. Revista Brasileira de Parasitologia Veterinária 22(1): 136-142. Disponível em: $<$ https://www.researchgate.net/profile/Wilson_Koller/publication /236089935_Mechanisms_of_pyrethroid_resistance_in_Haematobia_irritans_(Muscidae)_f rom_Mato_Grosso_do_Sul_state_Brazil/links/0046352696f7ca709d000000.pdf >. Acesso em: 10 jan. 2016. doi $10.1590 / S 1984-29612013005000016$.

Balbinot Junior, A.; Moraes, A., Veiga, M.; Pelissari, A.; Dieckow, J. 2009. Integração lavoura-pecuária: intensificação de uso de áreas agrícolas. Ciência Rural (39): 1925-1933. Disponível em: <http://www.scielo.br/pdf/cr/2009nahead/a229cr838.pdf>. Acesso em: 27 fev. 2016.

Belo, M.A.; Prado, R.J.E; Soares, E.V; Souza, M.L; Mota, D.C.; Giamlourenço, F.T.; Gírio, S.M.T. 2012 Eficácia de diferentes formulações no controle da mosca haematobia irritans em bovinos naturalmente infestados. Bioscience Journal 28(2): 245-250. Disponível em: 
<http://www.seer.ufu.br/index.php/biosciencejournal/article/viewFile/7373/8484>. Acesso em: 23 abr. 2016.

Bianchin, I.; Koller, W.W.; Detmann, E. 2006. Sazonalidade de Haematobia irritans no Brasil Central. Pesquisa Veterinária Brasileira 26(2): 79-86. Disponível em: <http://www.scielo.br/scielo.php?script=sci_arttext\&pid=S0100736X2006000200004\&lang=pt>. Acesso em: 23 abr. 2016. doi: 10.1590/S0100736X2006000200004

Bianchin, I; Alves, R.G. 2002. Mosca-dos-chifres, Haematobia irritans: comportamento e danos em vacas e bezerros Nelore antes da desmama. Pesquisa Veterinária Brasileira, 22(3): 109-113. Disponível em: <http://www.scielo.br/pdf/pvb/v22n3/12474.pdf>. Acesso em: 23 abr. 2016. doi: 10.1590/S0100-736X2002000300004

Bianchin, I.; Koller, W.W.; Oliveira Alves de, R.G.; Detmann, E. 2004. Efeito da moscados-chifres, Haematobia irritans (L.) (Diptera: Muscidae), no ganho de peso de bovinos Nelore. Ciência Rural 34(3): 885-890. Disponível em <http://dx.doi.org/10.1590/S0103$84782004000300035>$. Acesso em: 15 jan. 2016.

Brito, L.G.; Borja, M.E.G.; Oliveira, C. M.; Netto S.G F. 2005. Mosca-dos-chifres: aspectos bio-ecológicos, importância econômica, interações parasito-hospedeiro e controle. (Comunicado Técnico,1) Embrapa Rondônia, Porto Velho, RO. Disponível em: <http://ainfo.cnptia.embrapa.br/digital/bitstream/item/24807/1/Cot302-mosca-doschifres.pdf>. Acesso em: 22 abr. 2016.

Bruhn, F.R P.; Lopes, M.A.; Perazza, C.A.; Demeu, F.A.; dos Santos, G.; Neto, A.F.; Zeviani M.A.; Guimarães, A.M. 2013. Eficiência técnica e econômica da aplicação de diferentes anti-helmínticos em fêmeas da raça holandesa na fase de recria durante o outono inverno de 2009. Acta Tecnológica 7(2): 25-30. Disponível em:

<file:///C:/Users/User/ Downloads/138-839-1-PB\%20(3).pdf>. Acesso em: 22 abr. 2016.

Cicote, C.A.R.; Stort, L.A.; Neves, M. F. 2009. Onthophagus Gazella: Alternativa para controle da Haematobia irritans e recuperação de pastagem. Revista Científica Eletrônica de Medicina Veterinária 7(12). Disponível em:

$<\mathrm{http}$ ://faef.revista.inf.br/imagens_arquivos/arquivos_destaque/UNKAKeQNMmclOfl_2013 -6-21-12-1-1.pdf>. Acesso em: 12 jan. 2016.

Grisi, L.; Leite C.R.; Martins S.R.J.; Barros M.T.A.; Andreotti R.; Cançado D.H.P.; León P.A.A.; Pereira B.J.; Villela S.H. 2014. Reassessment of the potential economic impact of cattle parasites in Brazil. Revista Brasileira de Parasitologia Veterinária 23(2): 150-156. Disponível em: <http://www.scielo.br/scielo.php?script=sci_arttext\&pid=S198429612014000200150\&lang=pt>. Acesso em: 23 abr. 2016. doi: 10.1590/S198429612014042

Honer, M.R.; Bianchin, I.; Gomes, A. 1990. Mosca dos chifres: histórico, biologia e controle. (Circular Técnica, 45). Embrapa-CNPGC, Campo Grande, Mato Grosso do Sul, Brasil. Disponível em: <http://ainfo.cnptia.embrapa.br/digital/bitstream/item/104716/1/Mosca-dos-chifres.pdf>. Acesso em: 23 abr. 2016. 
Honer, M.R.; Gomes, A. 1990. O manejo integrado de mosca dos chifres, berne e carrapato em gado de corte. (Circular Técnica, 22). Embrapa-CNPGC, Campo Grande, Mato Grosso do Sul, Brasil. Disponível em:

$<$ http://www.sidalc.net/cgibin/wxis.exe/?IsisScript=ACERVO.

xis\&method=post\&formato $=2 \&$ cantidad $=1$ \&expresion $=m f n=038766>$. Acesso em: 23 abr. 2016.

Instituto Brasileiro Geografia e Estatística [IBGE]. 2014. Diretoria de pesquisas, coordenação de Agropecuária, Pesquisa da Pecuária Municipal. Disponível em:

<http://censo2010.ibge.gov.br/noticiascenso.html?view=noticia\&id=1 \&idnoticia=3006\&bus $\mathrm{ca}=1 \& \mathrm{t}=\mathrm{ppm}-2014$-rebanho-bovino-alcanca-212-3-milhoes-cabecas $>$. Acesso em: 22 abr. 2016.

Lopes, M.A.; dos Santos, G.; Neto, A.F.; Lopes, L.M.F.; Demeu, F.A.; Resende, B.L. 2012. Resultados econômicos de um sistema de produção de leite no município de ItutingaMG. Boletim de Indústria Animal 69(1): 23-31. Disponível em:

<http://revistas.bvsvet.org.br/bia/article/download/7156/7382>. Acesso em: 12 abr. 2016.

Lopes, M.A.; Carvalho, F. de M. 2002. Custo de produção do gado de corte. UFLA. Lavras, Minas Gerais, Brasil. Disponível em:

$<$ http://livraria.editora.ufla.br/upload/boletim/tecnico/boletim-tecnico-47.pdf>. Acesso em: 23 abr. 2016.

Paulino, M. F.; Detmann, E.; Silva G.A.; Almeida M.A.; Márquez C.E.D.; Moreno S.P.D.; Moura H.F.; Cardenas G.E.; Lima C.A.J.; Martins S.L.; Manso R.M.; Ortega M.E.R.; Lopes A.S.; Carvalho V.V. 2014. Bovinocultura otimizada. p. 139-164. In: Simpósio de produção de gado de corte, 9. Simpósio internacional de produção de gado de corte, 5. 2014. UFV. Viçosa, Minas Gerais, Brasil.

Sant Anna, F.B. 2007. Rola Bosta africano: um aliado no controle da mosca dos chifres. Disponível em: <http://www.agrosoft.org.br/agropag/26883.htm>. Acesso em: 11/04/2016.

Soutello, R.V.G. de. 2001. Influência do parasitismo e da suplementação no desenvolvimento ponderal de novilhos mestiços Angus-Nelore e da raça Guzerá. Disponível em:

<http://repositorio.unesp.br/bitstream/handle/11449/98643/soutello_rvg_me_ilha.pdf?sequ ence=1>. Acesso em: 23 abr. 2016.

Steelman, C.D.; Brown, A.H.; Gbur, E.E.; Tolley, G. 1991. Interactive response of the horn fly (Diptera: Muscidae) and selected breeds of beef cattle. Journal of economic entomology 84(4): 1275-1282. Disponível em: <https://jee.oxfordjournals.org/content/84/4/1275>. Acesso em: 10 abr. 2016.

Stotzer, E.S; Lopes L.B.; Eckstein, C.; Moraes, M.C.M.M.; Rodrigues, D.S.; Bastianetto, E. 2014. Impacto econômico das doenças parasitárias na pecuária. Revista Brasileira de Higiene e Sanidade Animal 8(3):198-221. Disponível em:

<http://www.higieneanimal.ufc.br/seer/index.php/higieneanimal/article/view/195/585> Acesso em: 22 abr. 2016. doi: 10.5935/1981-2965.20140128

Valério, J R; Guimarães, J.H. 1983. Sobre a ocorrência de uma nova praga, Haematobia irritans (L.)(Diptera, Muscidae), no Brasil. Revista Brasileira de Zoologia 1(4) : 417-418. Disponível em: <http://www.scielo.br/scielo.php?pid=S0101-817519820004000 
02\&script=sci_abstract\&tlng=es>. Acesso em: 23 abr. 2016. doi:

$<$ http://dx.doi.org/10.1590/S0101-81751982000400002>

Ziliotto, M.R.; Silveira C.; Camargo E.M.; Motta V.E.M.; Priesnitz Filho, W. 2010.

Comparação do Custo de Produção de Bovinocultura de Corte: Pasto versus

Confinamento. p. 1-12. In: VII SE-GeT - Simpósio de Excelência em Gestão e Tecnologia. Disponível em:

<http://correio.aedb.br/seget/artigos10/367_Artigo\%20SEGET\%20MEC.pdf>. Acesso em: 23 abr. 2016. 